\title{
In situ preparation of mixed anhydrides containing the trifluoroacetyl moiety. Application to the esterification of cholesterol and phenol
}

\author{
Sara Montiel-Smith, ${ }^{1}{ }^{1}$ Socorro Meza-Reyes, ${ }^{1}$ Omar Viñas-Bravo, ${ }^{1}$ \\ María A. Fernández-Herrera, ${ }^{1}$ Roxana Martínez-Pascual, ${ }^{1}$ Jesús Sandoval-Ramírez,*1 \\ Adriadna Fuente, ${ }^{2}$ Mayra Reyes, ${ }^{2}$ and José A. Ruiz ${ }^{2}$ \\ ${ }^{1}$ Facultad de Ciencias Químicas, Benemérita Universidad Autónoma de Puebla, C.U. 72570 \\ Puebla, Pue., México \\ ${ }^{2}$ Centro de Química Farmacéutica, Apartado postal 16042, Ciudad de La Habana, Cuba \\ E-mail: smontiel@siu.buap.mx
}

\section{Designated for Commemorative Issue in Honor of Prof. Eusebio Juaristi on his $55^{\text {th }}$ Anniversary \\ (received 24 Mar 05; accepted 24 Apr 05; published on the web 25 Apr 05)}

\begin{abstract}
This paper reports an easy characterization of carboxylic-trifluoroacetic mixed anhydrides by ${ }^{1} \mathrm{H}$ and ${ }^{13} \mathrm{C}$ NMR, and gives evidence that mixed anhydrides are produced instantaneously, at room temperature, from a carboxylic acid and trifluoroacetic anhydride and even from carboxylic anhydrides and trifluoroacetic acid. The esterification of phenol with the carboxylictrifluoroacetic anhydrides is highly chemoselective, and affords pure products, in quantitative yields, and in the case of cholesterol, some cholesteryl trifluoroacetate is also produced.
\end{abstract}

Keywords: Carboxylic-trifluoroacetic mixed anhydrides, esterification

\section{Introduction}

Esterification is a common procedure, widely reviewed, ${ }^{1}$ to transform carboxylic acids or their derivatives into an ester functional group, to protect alcohols or to achieve molecular features. Many esterification methodologies have been developed employing an additional Lewis acid catalyst, ${ }^{2}$ and among them the transesterification is used to attain selectivity, to improve yields, etc., through an easier way to attach an acyl group to a hydroxyl group. Besides those methodologies new ones are always desirable. In transesterification, a great problem arises from equilibration and to avoid that, sometimes a huge excess of one of the reactants is used and one 
of the product is constantly drained from the reaction. A particular transesterification methodology, which uses mixed anhydrides, has obtained a new impulse recently. The mixed anhydrides have been planned to contain a sterically hindered part of an acid or a good leaving group in one half of the molecule. ${ }^{3}$ A former mixed anhydride, the acetic-trifluoroacetic anhydride, has been used without purification because of its sensitiveness to moisture, tendency to disproportionate, and difficulty to establish the end point of its preparation. ${ }^{4}$

\section{Results and Discussion}

The formation of mixed anhydrides has been claimed through microanalysis, IR spectra, depressions of the freezing point, saponification equivalents, and of course, by the obtained acylated products. The common procedure to prepare mixed carboxylic-trifluoroacetic anhydrides is to heat a mixture of several equivalents of the carboxylic acid and trifluoroacetic anhydride (TFAA) during minutes to hours. ${ }^{5}$ A metathetical alternative, from silver trifluoroacetate and an acid chloride is also available. ${ }^{6}$ The end point of the preparation of the mixed anhydride has not been clearly defined, so when using heating, the reactions usually become dark and some materials are decomposed. We report herein an easy characterization of carboxylic-trifluoroacetic mixed anhydrides by ${ }^{1} \mathrm{H}$ and ${ }^{13} \mathrm{C}$ NMR.

When 1.2 mole of acetic acid is combined with 1.0 mole of TFAA a clear solution containing the acetic-trifluoroacetic anhydride (ATFAA) is formed in few seconds, as deduced by ${ }^{1} \mathrm{H}$ - and ${ }^{13} \mathrm{C}$-NMR spectra. In ${ }^{1} \mathrm{H}$-NMR three methyl singlets are immediately observed in the region around $2 \mathrm{ppm}$ : one for acetic acid (2.19 ppm), a second for $\mathrm{Ac}_{2} \mathrm{O}$ (2.27 ppm), and a third for ATFAA (2.38 ppm). In the ${ }^{13} \mathrm{C}$-NMR spectrum four signals are shown in the region for anhydride and acid carbonyls (Equation 1): ATFAA (s, 163.1, and q, $152.5 \mathrm{ppm}$ ), acetic anhydride (168.4 ppm), trifluoroacetic acid (q, $160.5 \mathrm{ppm})$ and acetic acid (179.8 ppm). Typical signals for ATFAA and trifluoroacetic acid are obtained due to their C-F couplings: ATFAA presents two coupling values: $45.5 \mathrm{~Hz}$ (for $\mathrm{C}$ of carbonyl) and $284.8 \mathrm{~Hz}$ (for $\mathrm{CF}_{3}$ ), and TFA other two: $43.3 \mathrm{~Hz}$ (for $\mathrm{C}$ of carbonyl) and $283.7 \mathrm{~Hz}$. (for $\mathrm{CF}_{3}$ ). The chemical shifts for all signals isolated compounds and for their signals in mixtures, as in the above reactions, have a slight variation. The excess of acetic acid and the produced trifluoroacetic acid can be eliminated by distillation, although some ATFAA is lost.

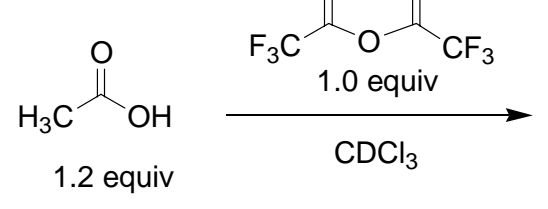

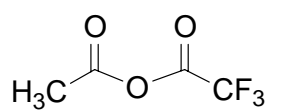

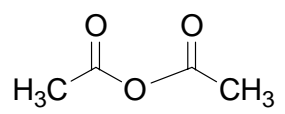

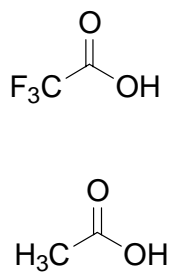

\section{Equation 1}


Following the procedure above described for ATFAA, other carboxylic-trifluoroacetic anhydrides were prepared. Data for some mixed anhydrides and for their starting carboxylic acids are presented in tables 1 and 2 ; in all reactions, yields were quantitative. $\mathrm{In}{ }^{1} \mathrm{H}-$ and ${ }^{13} \mathrm{C}-$ NMR spectra no traces of signals for protons or carbonyls groups from the starting acids were detected in the reaction crude. The formation of the mixed anhydrides occurred in a fast way even when the hindered pivalic acid or the aromatic benzoic acid was used.

Table 1. ${ }^{1} \mathrm{H}-\mathrm{NMR}$ Chemical shifts for the mixed anhydrides $\mathbf{1}-\mathbf{7}$, and their corresponding carboxylic acids

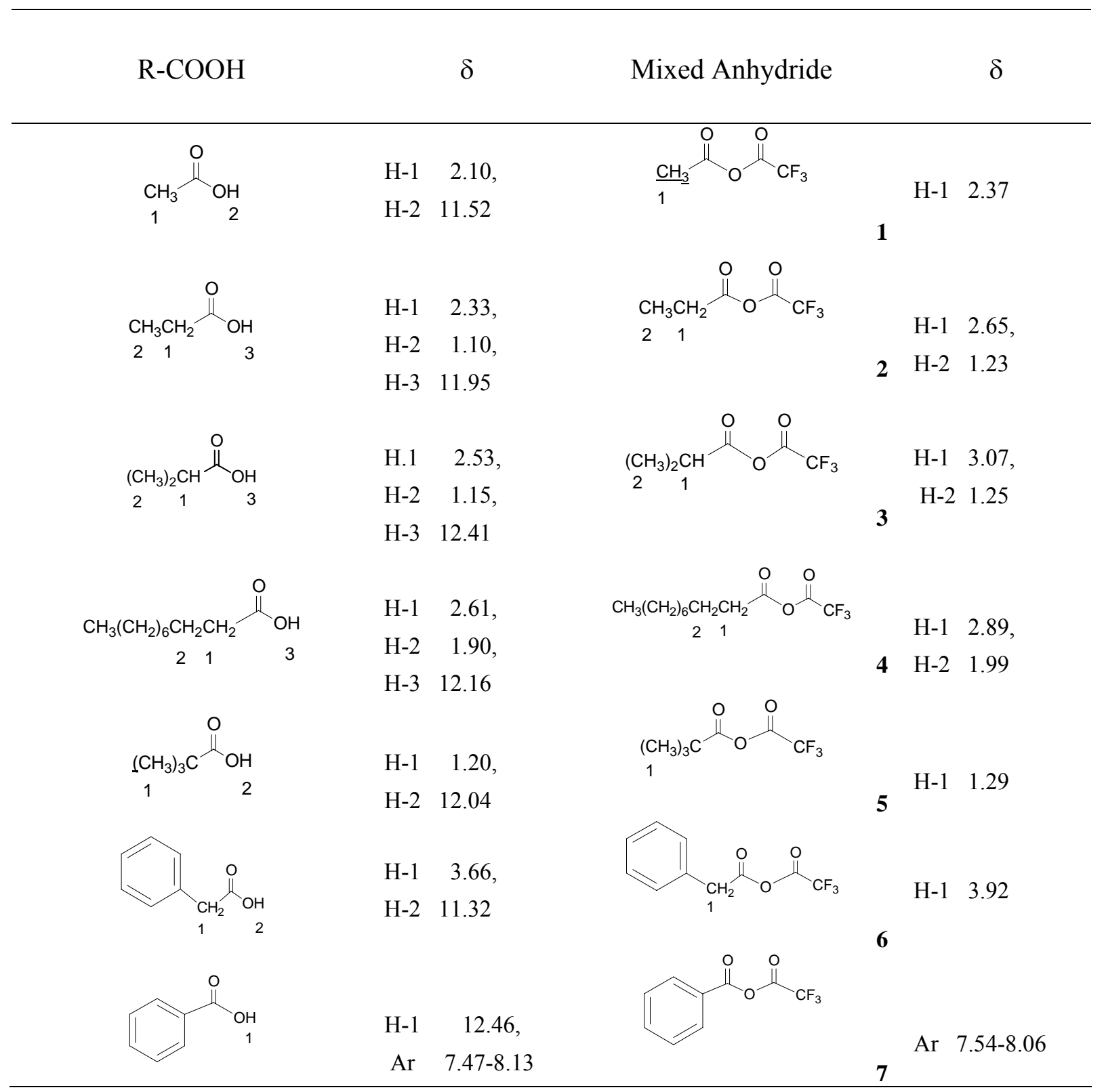


The esterification reaction of cholesterol with ATFAA was carried out quantitatively using $1 \mathrm{mmol}$ of substrate and $10 \mathrm{mmol}$ of mixed anhydride and for phenol the relation was 1:5, at room-temperature. In all cases, reactions of carboxylic-trifluoroacetic mixed anhydrides with cholesterol conduced to a small quantity of the cholesteryl trifluoroacetate $(5-12 \%)$. In the case of esterification of phenol, no trifluoroacetate was produced, indicating a high chemoselectivity of the alcohol group in phenol (Table 3). If the esterification reaction is runned out at $50{ }^{\circ} \mathrm{C}$ (the use of high temperature has been recommended in the literature ${ }^{4 a}$ ), the chemoselectivity is lost directing to $56 \%$ of cholesteryl acetate and $44 \%$ of cholesteryl trifluoroacetate, but the reaction turns brownish, indicating some decomposition.

Table 2. ${ }^{13} \mathrm{C}-\mathrm{NMR}$ Chemical shifts for $\mathbf{1}-\mathbf{7}$ mixed anhydrides, their corresponding carboxylic acids, TFAA, and trifluoroacetic acid

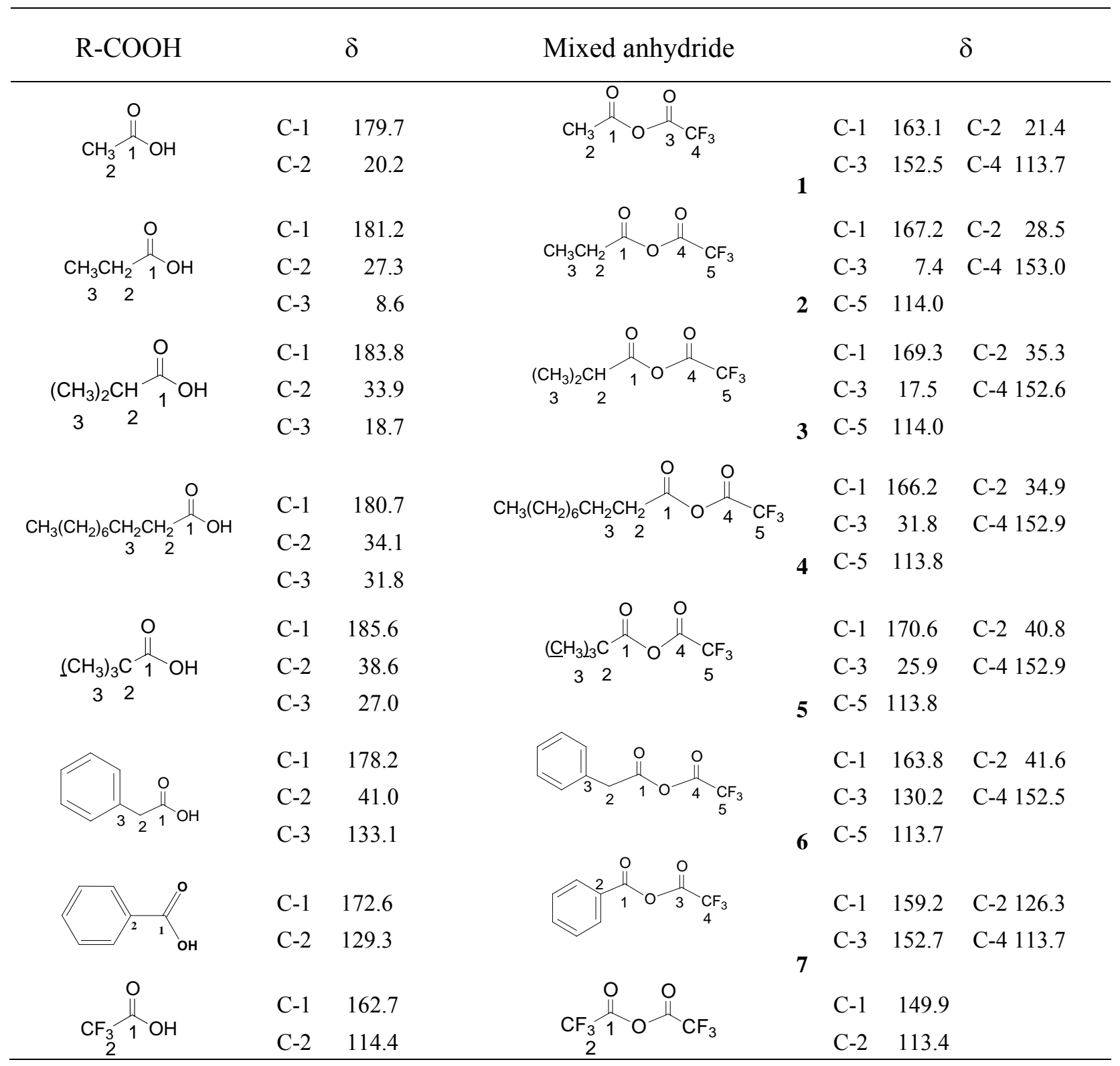


We have observed that carboxylic-trifluoroacetic mixed anhydrides can also be obtained using carboxylic anhydrides and trifluoroacetic acid; being this the first report on this new procedure. For example, ATFAA, propionic-trifluoroacetic anhydride and butyric-trifluoroacetic anhydride were prepared in a fast way $(5 \mathrm{~min}$ ) when equimolar quantities of both reagents were employed. In all those cases equilibria were produced being the mixed anhydrides present in 15$20 \%$. The equilibrated mixtures were used also for esterification reactions and it was found that the reactions proceeded in 5-10 min, indicating that the consumed mixed anhydride is rapidly reproduced. For example, esterification using $2 \mathrm{mmol}$ of $\mathrm{Ac}_{2} \mathrm{O}, 2 \mathrm{mmol}$ of $\mathrm{CF}_{3} \mathrm{COOH}$ and 1 mmol of phenol ( $1 \mathrm{~mL}$ of $\mathrm{CH}_{2} \mathrm{Cl}_{2}$ as co-solvent was used) directed to phenyl acetate in $1 \mathrm{~min}$, but when diluted phenol solutions $\left(0.01 \mathrm{M}\right.$ in $\left.\mathrm{CH}_{2} \mathrm{Cl}_{2}\right)$ containing only 0.05 equiv of $\mathrm{CF}_{3} \mathrm{COOH}$ were used, the reaction took $16 \mathrm{~h}$. When cholesterol was submitted to esterification under the above conditions, a mixture 4:1 of cholesteryl acetate and cholesteryl trifluoroacetate was obtained.

Table 3. Esterification products in reactions using mixed anhydrides

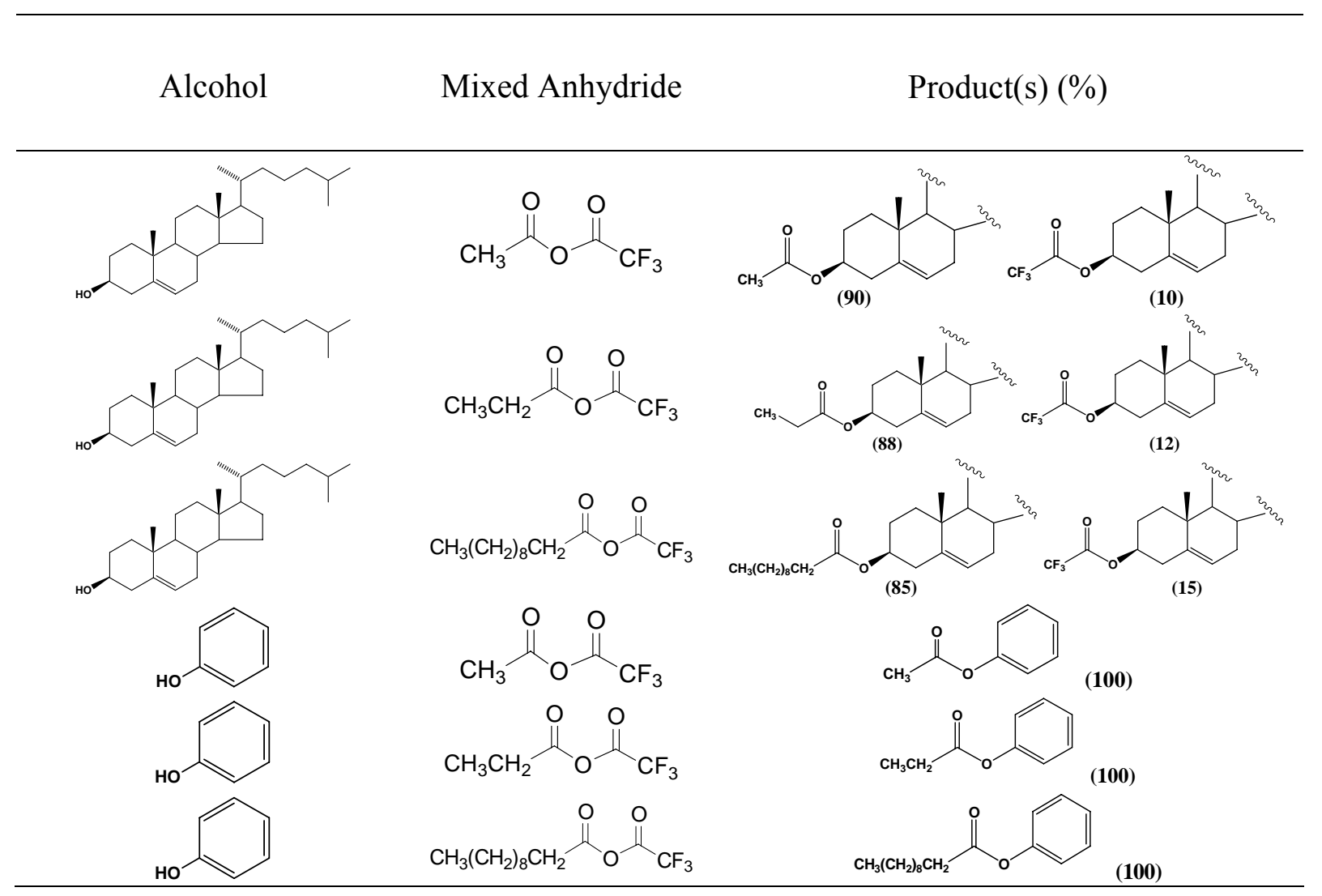

In summary, we describe a new method for the preparation of acetic-trifluoroacetic mixed anhydride from trifluoroacetic acid and acetic anhydride. Other mixed anhydrides containing the trifluoroacetyl moiety were prepared instantaneously from a carboxylic acid and trifluoroacetic anhydride; a different way to establish the end point of their formation reactions is reported, 
evidencing that it occurs quantitatively at room temperature. Cholesterol and phenol were rapidly esterified by the use of the mixed anhydrides, in high yields. This methodology could be applicated to other alcohols.

\section{Experimental Section}

General Procedures. Spectroscopy: NMR spectra were measured in $\mathrm{CDCl}_{3}$ on a Varian Mercury spectrometer at $400 \mathrm{MHz}$ for ${ }^{1} \mathrm{H}$ or $100 \mathrm{MHz}$ for ${ }^{13} \mathrm{C}$. Chemical shifts are expressed in ppm downfield from TMS. The optical rotations were determined on a Perkin Elmer 241 polarimeter, at room temperature. Melting points were obtained from a Mel-Temp apparatus and were not corrected.

\section{Typical procedure for esterification of phenol using a mixture of carboxylic anhydride and} trifluoroacetic acid

Acetic anhydride $(2.0 \mathrm{mmol})$ and trifluoroacetic acid $(2 \mathrm{mmol})$ were mixed, at room temperature, under argon, during 5 minutes. The formation of the mixed anhydride was established by NMR. Phenol ( $1 \mathrm{mmol}$ ) was dissolved in $1 \mathrm{~mL}$ of $\mathrm{CH}_{2} \mathrm{Cl}_{2}$ and added to the solution of ATFAA, at room temperature. Stirring continued until all the alcohol was consumed ( 5 minutes at most). At the end of the reaction, the crude was diluted by addition of $20 \mathrm{~mL}$ of $\mathrm{CH}_{2} \mathrm{Cl}_{2}$, washed with cold brine and iced $10 \%$ aq $\mathrm{NaHCO}_{3}$. The organic layer was dried with anh. $\mathrm{Na}_{2} \mathrm{SO}_{4}$, and evaporated under vacuum, to afford pure phenyl acetate.

\section{Typical procedure for esterification using a mixture of carboxylic acid and trifluoroacetic anhydride}

The formation of the mixed anhydrides was produced mixing $1.2 \mathrm{mmol}$ of corresponding carboxylic acid and $1.0 \mathrm{mmol}$ of the trifluoroacetic anhydride, at room temperature. This mixture was stirred under inert atmosphere during 30 seconds. A solution of cholesterol $(0.1 \mathrm{mmol})$ or phenol $(0.2 \mathrm{mmol})$ in $\mathrm{CH}_{2} \mathrm{Cl}_{2}(2 \mathrm{~mL})$, was incorporated at room temperature, and stirred until all the alcohol was consumed (3-5 minutes). At the end of the reaction, the crude was diluted with $20 \mathrm{ml}$ of $\mathrm{CH}_{2} \mathrm{Cl}_{2}$ and washed three times with cold brine, iced $10 \%$ aq $\mathrm{NaHCO}_{3}$. The organic layer was dried with anh. $\mathrm{Na}_{2} \mathrm{SO}_{4}$, and evaporated under vacuum, to dryness to afford pure esters.

\section{Compound characterization}

Cholesteryl acetate. mp $112-114{ }^{\circ} \mathrm{C}$ (E.P./AcOEt) (lit. $\left.{ }^{7} 114-115{ }^{\circ} \mathrm{C}\right) .[\alpha]_{\mathrm{D}}-45$ (c, 1.19 $\left.\mathrm{CHCl}_{3}\right)$ (lit. $\left.{ }^{7}[\alpha]_{\mathrm{D}}-47\right)$. 1H NMR: $5.4(1 \mathrm{H}, \mathrm{m}, \mathrm{H}-6), 4.6(1 \mathrm{H}, \mathrm{m}, \mathrm{H}-3), 2.03\left(3 \mathrm{H}, \mathrm{s}, \mathrm{CH}_{3}-\mathrm{AcO}\right)$, $1.02\left(3 \mathrm{H}, \mathrm{s}, \mathrm{CH}_{3}-19\right), 0.91\left(3 \mathrm{H}, \mathrm{d}, J_{20-21}=6.2 \mathrm{~Hz}, \mathrm{CH}_{3}-21\right), 0.86\left(3 \mathrm{H}, \mathrm{d}, J_{25-26}=6.6 \mathrm{~Hz}, \mathrm{CH}_{3}-26\right)$, $0.86\left(3 \mathrm{H}, \mathrm{d}, J_{25-27}=6.6 \mathrm{~Hz}, \mathrm{CH}_{3}-27\right) .{ }^{13} \mathrm{C} \mathrm{NMR}: 37.0(\mathrm{C}-1), 27.8$ (C-2), 73.9 (C-3), 38.1 (C-4), 139.4 (C-5), 122.5 (C-6), 31.9 (C-7), 31.9 (C-8), 50.0 (C-9), 36.6 (C-10), 21.1 (C-11), 39.5 (C- 
12), 42.3 (C-13), 57.7 (C-14), 24.3 (C-15), 28.3 (C-16), 56.1 (C-17), 11.9 (C-18), 19.4 (C-19), 35.8 (C-20), 18.8 (C-21), 36.2 (C-2), 23.9 (C-23), 39.7 (C-24), 28.1 (C-25), 22.9 (C-26), 22.6 (C27), 170.2 ( $\left.\mathrm{CH}_{3} \underline{\mathrm{COO}}\right), 21.5$ ( $\left.\underline{\mathrm{CH}}_{3} \mathrm{COO}\right)$.

Cholesteryl trifluoroacetate. $\mathrm{mp} 131-133{ }^{\circ} \mathrm{C}$ (E.P./AcOEt) (lit. $\left.{ }^{8} 134-136{ }^{\circ} \mathrm{C}, \mathrm{EtOH}\right) .[\alpha]_{\mathrm{D}}-41$ (c, $\left.1.05, \mathrm{CHCl}_{3}\right)\left(\right.$ lit. $\left.^{8}[\alpha]_{\mathrm{D}}-37\right) . \delta{ }^{1} \mathrm{H}: 5.42(1 \mathrm{H}, \mathrm{m}, \mathrm{H}-6), 4.80(1 \mathrm{H}, \mathrm{m}, \mathrm{H}-3), 1.05\left(3 \mathrm{H}, \mathrm{s}, \mathrm{CH}_{3}-19\right)$, $0.93\left(3 \mathrm{H}, \mathrm{d}, J_{20-21}=6.3 \mathrm{~Hz}, \mathrm{CH}_{3}-21\right), 0.87\left(6 \mathrm{H}, \mathrm{d}, J=6.6 \mathrm{~Hz}, 2 \mathrm{CH}_{3}-26,27\right), 0.69\left(3 \mathrm{H}, \mathrm{s}, \mathrm{CH}_{3}-18\right) . \delta$ ${ }^{13} \mathrm{C}: 36.8$ (C-1), 27.3 (C-2), 78.6 (C-3), 37.5 (C-4), 138.5 (C-5), 123.8 (C-6), 31.9 (C-7), 31.8 (C8), 50.0 (C-9), 36.5 (C-10), 21.1 (C-11), 39.7 (C-12), 42.3 (C-13), 56.7 (C-14), 24.3 (C-15), 28.3 (C-16), 56.3 (C-17), 11.8 (C-18), 11.8 (C-18), 19.2 (C-19), 35.9 (C-20), 18.7 (C-21), 36.3 (C22), 23.9 (C-23), 39.6 (C-24), 28.0 (C-25), 22.6 (C-26), 22.8 (C-27), 156.9 (c, J=41.7 Hz, $\mathrm{CF}_{3} \underline{\mathrm{COO}}-3$ ), 114.6 (c, J=285.9 Hz, $\left.\mathrm{CF}_{3} \mathrm{COO}-3\right)$.

Cholesteryl propionate. mp $116{ }^{\circ} \mathrm{C}$ (E.P./AcOEt) (lit. $\left.{ }^{7} 114{ }^{\circ} \mathrm{C}\right) .[\alpha]_{\mathrm{D}}-42$ (c, $\left.1.07 \mathrm{CHCl}_{3}\right)\left(\right.$ lit. $^{7}$ $\left.[\alpha]_{\mathrm{D}}-41\right) . \delta{ }^{1} \mathrm{H}: 5.35(1 \mathrm{H}, \mathrm{m}, \mathrm{H}-6), 4.60(1 \mathrm{H}, \mathrm{m}, \mathrm{H}-3), 2.29\left(2 \mathrm{H}, \mathrm{c}, J=7.5 \mathrm{~Hz}, \mathrm{CH}_{3} \underline{\mathrm{H}}_{2} \mathrm{COO}-3\right)$, $1.13\left(3 \mathrm{H}, \mathrm{t}, J=7.5 \mathrm{~Hz}, \mathrm{CH}_{3} \mathrm{CH}_{2} \mathrm{COO}-3\right), \quad 1.02\left(3 \mathrm{H}, \mathrm{s}, \mathrm{CH}_{3}-19\right), 0.91\left(3 \mathrm{H}, \mathrm{d}, J_{21-20}=6.5 \mathrm{~Hz} \mathrm{CH} 3^{-}\right.$ 21), $0.86\left(6 \mathrm{H}, \mathrm{d}, 2 \mathrm{CH}_{3}-26,27\right), 0.67\left(3 \mathrm{H}, \mathrm{s}, \mathrm{CH}_{3}-18\right) . \delta{ }^{13} \mathrm{C}: 37.0$ (C-1), 27.9 (C-2), 73.7 (C-3), 38.2 (C-4), 139.5 (C-5), 122.4 (C-6), 31.9 (C-7), 31.9 (C-8), 50.0 (C-9), 36.6 (C-10), 21.1 (C11), 39.8 (C-12), 42.3 (C-13), 56.7 (C-14), 24.3 (C-15), 28.3 (C-16), 56.1 (C-17), 11.9 (C-18), 19.4 (C-19), 35.8 (C-20), 18.8 (C-21), 36.2 (C-22), 23.9 (C-23), 39.5 (C-24), 28.1 (C-25), 22.6 (C-26), 22.9 (C-27), $173.6\left(\mathrm{CH}_{3} \mathrm{CH}_{2} \underline{\mathrm{COO}}-3\right), 28.0\left(\mathrm{CH}_{3} \underline{\mathrm{CH}}_{2} \mathrm{COO}-3\right), 9.3\left(\mathrm{CH}_{3} \underline{\mathrm{CH}}_{2} \mathrm{COO}-3\right)$.

Cholesteryl decanoate. mp $78-79^{\circ} \mathrm{C}$ (E.P./AcOEt) (lit. $\left.{ }^{7} 80-81^{\circ} \mathrm{C}\right)$. $[\alpha]_{\mathrm{D}}-32$ (c, $\left.1.04 \mathrm{CHCl}_{3}\right)\left(\right.$ lit. $^{7}$ $\left.[\alpha]_{\mathrm{D}}-27\right) . \delta{ }^{1} \mathrm{H}: 5.37(1 \mathrm{H}, \mathrm{m}, \mathrm{H}-6), 4.61(1 \mathrm{H}, \mathrm{m}, \mathrm{H}-3), 2.27\left(2 \mathrm{H}, \mathrm{t}, \mathrm{CH}_{3}\left(\mathrm{CH}_{2}\right)_{7} \mathrm{C}_{2}{ }_{2} \mathrm{COO}-3\right), 1.03$ $\left(3 \mathrm{H}, \mathrm{s}, \mathrm{CH}_{3}-19\right), 0,93\left(3 \mathrm{H}, \mathrm{d}, J_{21-20}=6.8 \mathrm{~Hz} \mathrm{CH}-21\right), 0.87\left(6 \mathrm{H}, \mathrm{d}, J=6.6 \mathrm{~Hz}, 2 \mathrm{CH}_{3}-26,27\right), 0.69$ $\left(3 \mathrm{H}, \mathrm{s}, \mathrm{CH}_{3}-18\right) . \delta{ }^{13} \mathrm{C}: 37.0(\mathrm{C}-1), 27.8(\mathrm{C}-2), 73.6(\mathrm{C}-3), 38.1(\mathrm{C}-4), 139.7(\mathrm{C}-5), 122.5(\mathrm{C}-6)$, 31.8 (C-7), 31.8 (C-8), 50.0 (C-9), 36.6 (C-10), 21.0 (C-11), 39.7 (C-12), 42.3 (C-13), 56.6 (C14), 24.2 (C-15), 28.2 (C-16), 56.1 (C-17), 11.8 (C-18), 19.3 (C-19), 35.7 (C-20), 18.7 (C-21), 36.1 (C-22), 23.8 (C-23), 39.5 (C-24), 28.0 (C-25), 22.5 (C-26), 22.8 (C-27), 34.7

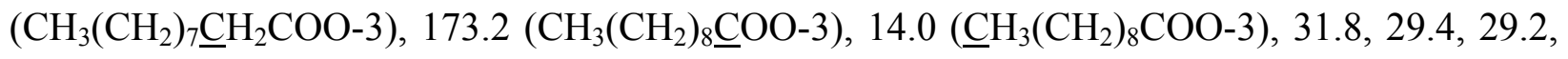
29.1, 25.0, $22.6\left(\mathrm{CH}_{3}\left(\underline{\mathrm{CH}}_{2}\right)_{7} \mathrm{CH}_{2} \mathrm{COO}-3\right)$.

Phenyl acetate. bp $188-190{ }^{\circ} \mathrm{C}, 598 \mathrm{~mm} \mathrm{Hg}$ (lit. $\left.{ }^{9} 195-197{ }^{\circ} \mathrm{C}, 760 \mathrm{~mm} \mathrm{Hg}\right) . \delta{ }^{1} \mathrm{H}: 2.25$ (3H, s, $\mathrm{CH}_{3}$ ), 7.05 (2H, dddd, $J=8.4,2.1,1.5,0.6 \mathrm{~Hz}, \mathrm{H}$-ortho), 7.33 (2H, dddd, $J=8.4,8.1,1.9,0.9$ $\mathrm{Hz}$, H-meta), $7.18\left(1 \mathrm{H}\right.$, dddd, $J_{1}=J_{2}=8.1, J_{3}=J_{4}=2.1 \mathrm{~Hz}, \mathrm{H}-$ para $) . \delta{ }^{13} \mathrm{C}: 125.5$ (C-para), 129.2 (C-meta), 121.3 (C-ortho), 150.4 (C-ipso), 169.1 (C-1), 21.1 (C-2).

Phenyl propionate. bp 203-205 ${ }^{\circ} \mathrm{C}, 598 \mathrm{~mm} \mathrm{Hg}$ (lit. ${ }^{9} 211{ }^{\circ} \mathrm{C}, 760 \mathrm{~mm} \mathrm{Hg}$ ). $\delta{ }^{1} \mathrm{H}: 2.57$ (2H, q, $J$ $\left.=7.6 \mathrm{~Hz},-\mathrm{CH}_{2}-\mathrm{CH}_{3}\right), 1.25\left(3 \mathrm{H}, \mathrm{t}, J=7.9 \mathrm{~Hz},-\mathrm{CH}_{2}-\mathrm{CH}_{3}\right), 7.05(2 \mathrm{H}$, dddd, $J=8.6,3.6,3.9,1.0$ $\mathrm{Hz}$, H-ortho), 7.34 ( $2 \mathrm{H}$, dddd, $J=8.6,8.4,3.2,0.8 \mathrm{~Hz}, \mathrm{H}-m e t a), 7.19$ (1H, dddd, $J_{1}=J_{2}=8.4$, $J_{3}=J_{4}=3.6 \mathrm{~Hz}, \mathrm{H}$-para). $\delta{ }^{13} \mathrm{C}: 125.5$ (C-para), 129.2 (C-meta), 121.4 (C-ortho), 150.5 (C-ipso), 172.7 (C-1), 27.8 (C-2), 9.2 (C-3).

Phenyl decanoate. ${ }^{10}$ bp $298-300{ }^{\circ} \mathrm{C}(\mathrm{dec}) \delta{ }^{1} \mathrm{H}: 2.54\left(2 \mathrm{H}, \mathrm{t}, J=7.5 \mathrm{~Hz}, \mathrm{CO}-\mathrm{CH}_{2}\right), 1.75(2 \mathrm{H}, \mathrm{q}, J$ $\left.=7.5 \mathrm{~Hz}, \mathrm{CH}_{2}-3\right), 1.28\left(12 \mathrm{H}, \mathrm{m}, \mathrm{CH}_{2}-4,5,6,7,8\right.$, and 9), $0.88\left(3 \mathrm{H}, \mathrm{t}, J=6.8 \mathrm{~Hz},-\mathrm{CH}_{2}-\mathrm{CH}_{3}\right)$, $7.05(2 \mathrm{H}$, dddd, $J=8.7,2.1,1.9,1.0 \mathrm{~Hz}, \mathrm{H}$-ortho $), 7.35$ (2H, dddd, $J=8.7,7.5,2.3,0.9 \mathrm{~Hz}, \mathrm{H}-$ 
meta), $7.20\left(1 \mathrm{H}\right.$, dddd, $J_{1}=J_{2}=7.5, J_{3}=J_{4}=2.1 \mathrm{~Hz}, \mathrm{H}-$ para $) . \delta{ }^{13} \mathrm{C}: 125.5$ (C-para), 129.2 (Cmeta), 121.4 (C-ortho), 150.6 (C-ipso), 172.1 (C-1), 34.5 (C-2), 25.1 (C-3), 29.5 (C-4), 29.4 (C5), 29.2 (C-6), 29.4 (C-7), 32.0 (C-8), 22.8 (C-9), 14.2 (C-10).

\section{Supplementary information}

Selected ${ }^{1} \mathrm{H}$ and ${ }^{13} \mathrm{C}$ NMR spectra for carboxylic-trifluoroacetic anhydrides and esters of cholesterol and phenol are included.

\section{Acknowledgements}

We are grateful to CONACyT, Mexico, for financial support via grant Bilateral J200-643 and the scholarships granted to O. V. B., M. A. F. H., R. M. P., and for stage of M. R.

\section{References}

1. (a) McOmie, J. F. W. Protective Groups in Organic Chemistry; Plenum Press: London, 1973. (b) Greene, T. W.; Wuts, P. G. M. Protective Groups in Organic Synthesis, $3^{\text {rd }}$ Edn.; John Wiley \& Sons: New York, 1999. (c) Kocienski, P. J. Protecting Groups, $2^{\text {nd }}$ Edn.; Georg Thieme Verlag: Stuttgart, 2000. (d) Otera, J. Esterification; Wiley-VCH GmbH \& Co. KgaA: Weinheim, 2003.

2. (a) Procopiou, P. A.; Baugh, S. P. D.; Flack, S. S.; Inglis, G. G. A. Chem. Commun. 1996, 2625. (b) Ishihara, K.; Kubota, M.; Kurihara, H.; Yamamoto, H. J. Org. Chem. 1996, 61, 4560. (c) Pansare, S. V.; Malusare, M. G.; Rai, A. N. Synth. Commun. 2000, 30, 2587. (d) Otera, J. Angew. Chem. Int. Ed. 2001, 40, 2044. (e) Xu, Q. H. X.; Chen, B. H.; Ma, Y. X. Synth. Commun. 2001, 31, 2113. (f) Das, B.; Venkataiah, B.; Madhusudhan, P. Synth. Commun. 2002, 32, 249. (g) Stefane, B.; Kocevar, M.; Polanc, S. Synth. Commun. 2002, 32, 1703. (h) Pan, W. B. ; Chang, F. R. ; Wei, L. M.; Wu, M. J.; Wu, Y. Ch. Tetrahedron Lett. 2003, 44, 331. (i) Otera, J. Acc. Chem. Res. 2004, 37, 288. (j) Martínez-Pascual, R.; ViñasBravo, O.; Meza-Reyes, S.; Iglesias-Arteaga, M. A.; Sandoval Ramírez, J. Synth. Commun. 2004, 34, 4591. (k) Chakraborti, A. K.; Gulhane, R. Synlett 2004, 627. (1) Oohashi, Y.; Fukumoto, K.; Mukaiyama, T. Chem. Lett. 2004, 33, 968. (m) Lu, K. C. L.; Hsieh, S. Y.; Patear, L. N.; Chen, C. T.; Lin, C. C. Tetrahedron 2004, 60, 8967. (n) De S. K. Tetrahedron Lett. 2004, 45, 2919. (o) Dandapani, S.; Curran, D. P. J. Org. Chem. 2004, 69, 8751.

3. (a) Goossen, L. J.; Ghosh, K. Eur. J. Org. Chem. 2002, 3254. (b) Shiina, I. Tetrahedron 2004, 60, 1587. (c) Goossen, L. J.; Döhring, A. Synlett 2004, 263. (d) Dauvergne, J.; Wellington, K.; Chibale, K. Tetrahedron Lett. 2004, 45, 43. 
4. (a) Bourne, E. J.; Stacey, M.; Tatlow, J. C.; Tedder, J. M. J. Chem. Soc. 1949, 2976. (b) Emmons, W. D.; McCallum, K. S.; Ferris, A. F. J. Am. Chem. Soc. 1953, 75, 6047. (c) Ranu, B. C.; Ghosh, K.; Jana, U. J. Org. Chem. 1996, 61, 9546.

5. (a) Stacey, M.; Bourne, E. J.; Tatlow, J. C.; Tedder, T. M. Nature 1949, 164, 705. (b) Morgan, P. W. J. Am. Chem. Soc. 1951, 73, 860. (c) Emmons, W. D.; McCallum, K. S.; Ferris, A. F. J. Am. Chem. Soc. 1953, 75, 6047.

6. Ferris, A. F.; Emmons, W. D. J. Am. Chem. Soc. 1953, 75, 232.

7. Dictionary of Steroids. Hill, R. A.; Makin, H. L. J.; Kirk, D. N.; Murphy, G. M. Eds. Chapman \& Hall: London, 1991.

8. Westphal, D.; Zbiral, E. Justus Liebigs Ann. Chem. 1975, 2038

9. Pollock, J. R. A.; Stevens, R. Dictionary of Organic Compounds; Eyre \& Spottiswoode, Publ. Ltd.: London, 1965

10. Erdmann, L.; Uhrich, K. E. Biomaterials 2000, 21, 1941. 